\title{
DIGITAL TERRAIN MODELS APPLIED TO THE C/I RATIO DETERMINATION IN FREQUENCY REUSE DESIGN
}

\author{
Narcís Cardona, Javier Martí, Miguel Ferrando \\ Departamento de Comunicaciones \\ Universidad Politécnica.Valencia (Spain)
}

\section{SUMMARY}

One of the main problems planning mobile cellular systems is to find the cochannel interference areas which affect a cell site or produced from a mobile receiver. The usual treatment of this problem consists in the adoption of a theoretical model to obtain the mean coverage or interference radio, ignoring possible ambiguity zones, "holes" of weak signal strength and cochannel interference areas due to the real environment. In this abstract we present a tool developed to plannify cellular systems based on the application of theoretical studies of propagation phenomena in urban areas to the real digital terrain models of a city. This permits the design of the cellular structure in an interactive way taking account of the real interference and ambiguity zones. Methods for the reduction of cochannel interference are also considered.

\section{THEORETICAL MODELS}

In the planning of mobile communication systems, such as urban mobile cellular telephony which cover a large number of users, often appears the need to calculate some restrictive parameters like interference level. Usually these parameters are approximated to a uniform behaviour or mean values into the same area. Obviously, one never treat to characterize the whole phenomena which constitute the urban area propagation, but two types of solutions are used: Approximated theoretical models of the mean performance in a concrete area or experimental models based on adding corrections obtained from measures made in a specific city. There are several studies about each phenomena in this type of propagation that results in models of reflection, fading and scattering over urban buildings and structures [1..6]. These models used to be validated by a statistical study of measurements taken in an extended zone.

Nevertheless, mobile telephony network planning al ways demands a more detailed treatment of interference between cells. This parameter constitutes the main limitation in frequency reuse design. In this case, the cell coverage area doesn't look like a circle or an hexagon and a high percentage of ambiguity zones appear in the cellular structure.

One possible way to reduce this uncertainty in the cellular systems design consists of applying the theoretical models which characterize the performance of the different urban structures into a digitalized model of the urban area.

\section{DIGITAL TERRAIN MODELS APPLICATION}

An application oriented to this kind of design has been developed on MATLAB (a numeric computation and data analysis software). At first, fictitious random city models were used, and some of the urban area propagation models from recent publications has been applied. [3..6]

MATLAB's facility to operate with arrays permits to calculate in a short time of process parameters like coverage of a base station, carrier to interference relation between cochannel cells, et cetera. This is a very useful and powerful tool for planning and designing this kind of networks because it permits automatic operations like:

-Find weak signal strength zones in the coverage area,

-Allocate the base stations at the best point according to the criteria adopted in each case ( $\mathrm{C} / \mathrm{I}$, for instance),

-Decide the best orientation of sectors in each cell,

-Modelling effect of the different structures on the propagation path separately or simultaneously.

In conclusion, it doesn't obtain radios but zones or "maps" of any parameter.

This method has been applied to a real digital model corresponding to the city of Valencia in the first step to plan the future GSM standard system and in the study to the enlargement of the actual TMA900 network.

\section{FEATURES}

The main features of the tool developed are shown in next figures. In the following examples $900 \mathrm{MHz}$ band was used and the receiver antenna height was fixed to $1.5 \mathrm{~m}$.

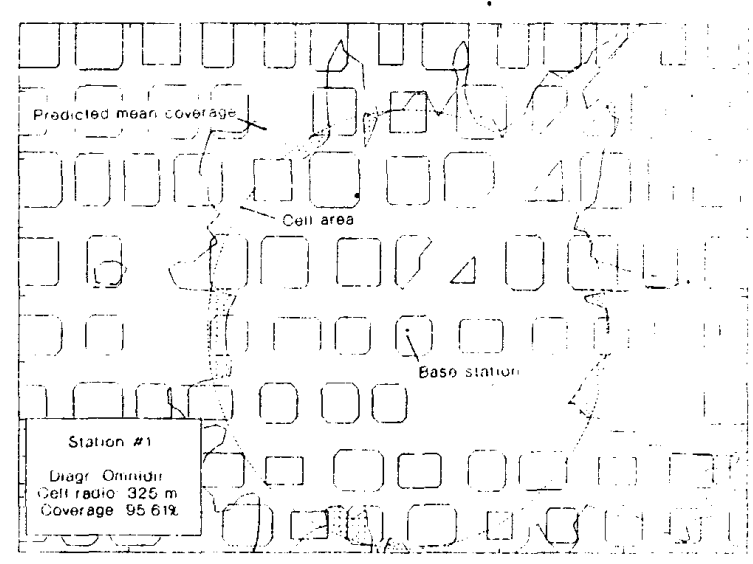

Figure 1. Coverage area in a microcell 


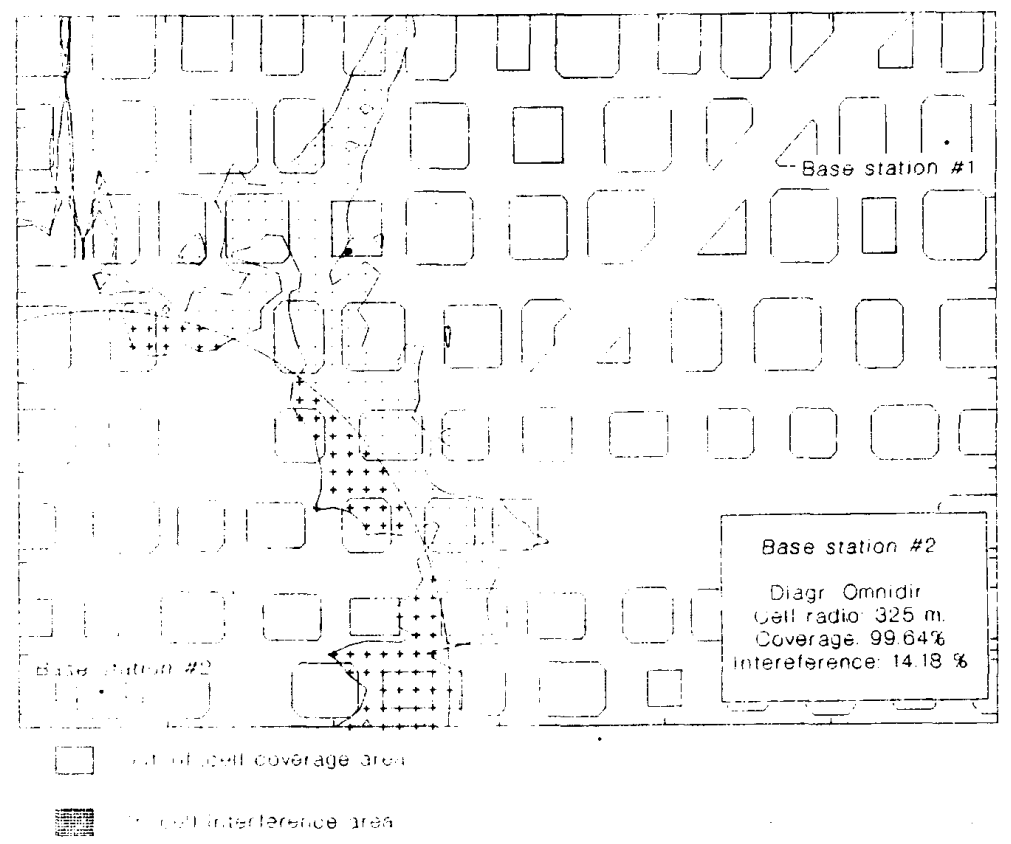

Figure 2. Enlargement of interference zone determination.

\section{Coverage}

Theoretical coverage zone can be calculated applying any propagation model. The program estimates the percentage of the area covered and, if the digital terrain model has enough resolution, holes of weak signal strength in the desired coverage zone can be detected. Figure 1 shows a result for a microcell designed to cover a circular zone ( 300 meters radio). Omnidirectional condition for the transmitter antenna has been adopted and the propagation model applied is taken from Walfish-Bertoni [3], which suppose scattering in urban buildings like the main contribution to the signal received at street level.

\section{Interference}

In this type of systems the frequency-reuse method is useful for increasing the efficiency of spectrum usage. This obviously results in cochannel interference between cells. With the tool developed, a "map" of interference zones can be obtained. Percentages of the cell coverage area affected by interferences are calculated. This percentage can be improved looking for the best base station allocations. In figure 2 the zones with a low carrier to interference ratio due to a near cochannel cell are shown. In this case, a 4_cell_reuse pattern is used, which suppose that distance between annel cells is about $3.5 R$ (where $R$ is the radio of each cell).

\section{Reduction of cochannel interference}

Diverse methods for the reduction of interference between cochannel cells has been implemented: use of directional antennas (each cell is divided into 3 or 6 sectors), lowering the antenna heights at the base station, tilting the antenna on the coverage pattern (a notch to the cochannel cell direction appears in the horizontal diagram) and using umbrella patterns.

The most effective of these methods is the sectorization of cells. In the tool presented, different antenna diagrams are used. These diagrams respond to a expression or could be a real measured behaviour. The automatic correction of the coverage and interference maps with the directive diagram allows to find the best orientation of the sectors in each cell. This function has been implemented and it takes into account the limits and conditions imposed by the type of cell pattern used.

Figures $4 \& 5$ show two steps in the optimum_sector_direction function. The antenna used here has a directive diagram, each cell is divided into 3 sectors and the 4_cell_reuse pattern is supposed. In figure 3 the usual orientation in this type of patterns is considered (being 4.1 radians in this step). Because the interfered area in cell \#2 is $15.43 \%$ of the sector coverage zone, the program looks for a different orientation finding an optimum of 4.28 radians which minimize the cochannel interference from cell \#1 (see figure 4). 


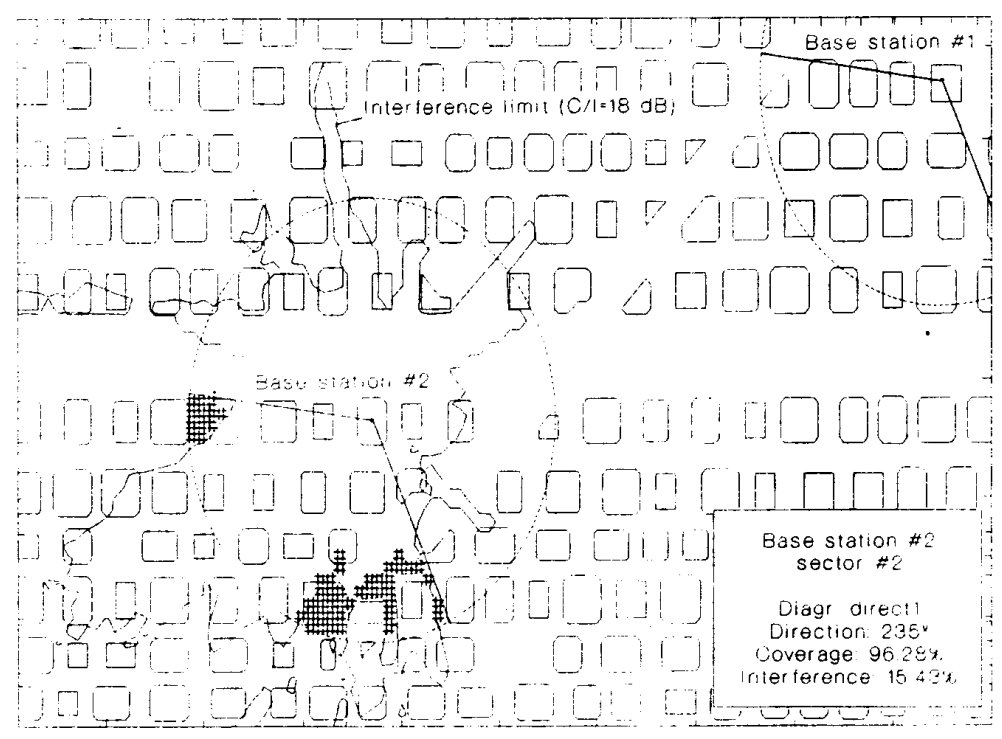

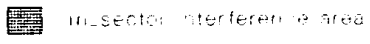

Figure 3. Oprimum sector direction (first step: 4.1 radians)

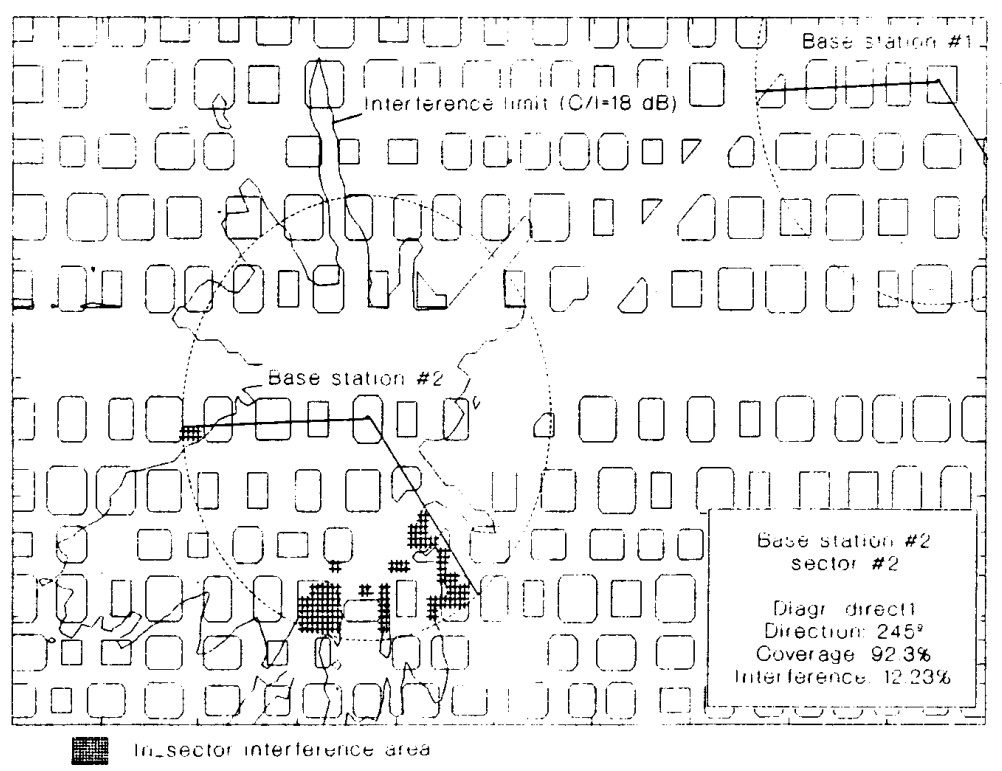

Figure 4. Optimum sector direction (last step: 4.28 radians)

2.2 .3 


\section{NEXT STUDIES}

Examples presented in this paper were made for a reduced area in order to mantain the legibility of the results. They respond to the firsts studies made on a '386 personal computer. Planning of wide areas or city networks requires the simultaneous treatment of a few base stations and the evaluation of parameters in a large number of points. A workstation version of the program is required in these cases.

The propagation model applied in the examples was simply. It just takes into account the limits of the buildings and their height. If reflections, trees, ground type or other factors must be considered, it will be convenient to manipulate this information into a Geographic Information System (GIS). The main advantage of GIS is the data base capability and easy storage of information in layers. Each layer respond to a parameter, could be treated separately and will add a correction to each point of the carrier to interference map.

\section{CONCLLSIONS}

A tool to plannify cellular systems using digital terrain models has been presented. The parameters of coverage $\&$ interference are calculated for each point of the model and the results are presented like maps of coverage and interference zones. Functions oriented to automatic improvement of the network structure (like reduction of cochannel interference) has been developed. Now this application is been implemented on a workstation. Among other options, this will permit the almost real time actualization of calculated parameter maps, being a powerful tool for interactive planning of mobile cellular systems and also could be used in other type of similar problems.

\section{REFERENCES}

[1] V. Graciáno . "Propagation correlations at $900 \mathrm{MHz}$ IEEE T-VT vol27 Nov 78 182-189

[2] Allsebrook, Parsons. "Mobile radio propagation in British cities at frequencies in the VHF and UHF bands" IEEE T-VT vol26 Nov 77 313-322

[3] Walfish, Bertoni. "A theoretical model of UHF propagation model in urban environments"; IEEE T-AP Dec 88 1788-1796

[4] Hoffmeyer J.A. "Measurement, modeling and simulation of digital LOS microwave channels..." IEEE T-Com vol39 Sep 91 1295-1305

[5] Yao, Sheikh. "Investigations into cochannel interference in microcellular mobile radio systems" IEEE T-VT vol41 May 92 114-123

[6] V. Erceg et Al. "Urban/suburban out-of-sight propagation modelling" IEEE Com-mag June 92 56-61

$$
2.2 .3
$$

